\title{
Does a core stabilization exercise program have a role on shoulder rehabilitation? A comparative study in young females
}

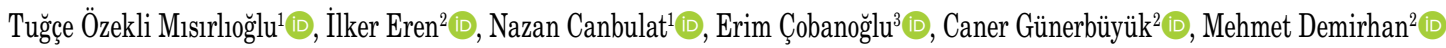 \\ ${ }^{1}$ Department of Physical Medicine and Rehabilitation, Koç University School of Medicine, İstanbul, Turkey \\ ${ }^{2}$ Department of Orthopaedics and Traumatology, Koç University School of Medicine, İstanbul, Turkey \\ ${ }^{3}$ Koç University School of Medicine, İstanbul, Turkey
}

Received: March 05, 2017 Accepted: January 29, 2018 Published online: August 16, 2018

\begin{abstract}
Objectives: This study aims to evaluate the effect of core stabilization exercises and to explore the immediate effect of core muscles-activated posture on shoulder maximal voluntary isometric contraction (MVIC) strength.

Patients and methods: Between November 2016 and January 2015, a total of 75 healthy female volunteers (mean age 25.36 years; range, 18 to 30 years) were included. Of these, 42 consecutive volunteers were assigned as the study group, while the remaining 33 volunteers were assigned as the control group. The study group completed a six-week core stabilization home-based exercise program. Two measurements were performed with six-week interval. A set of three repetitions for each shoulder side was performed by an electronic dynamometer under two conditions: core muscles relaxed and activated. Measurements were monitored real-time with a connected computer and recorded in Newton.

Results: The activation of core muscles during the measurement significantly decreased the MVIC values in both groups (p<0.05). The MVIC values significantly increased after home-based exercise program in both conditions $(\mathrm{p}<0.05)$.

Conclusion: Our study showed that six-week core stabilization exercise program had a significant positive effect on the shoulder MVIC strength. This result may support the use of core stabilization exercises in the early periods of shoulder rehabilitation when the shoulder muscle strengthening exercises are painful.
\end{abstract}

Keywords: Muscle strength dynamometer; rotator cuff; shoulder.

Improvement of the shoulder muscle strength is one of the major goals of shoulder rehabilitation. ${ }^{[1]}$ The appropriate mode of exercises for strengthening shoulder musculature are isometric, concentric, eccentric training, or open/closed chain activities. Upper extremity kinetic chain exercises have been integrated into the course of shoulder rehabilitation process in recent years. These exercises use functional movement patterns to facilitate scapular motion and, then, to strengthen scapular and rotator cuff musculature. ${ }^{[2]}$

The mechanical linkages of body segments which allow for the sequential transfer of forces and motions when performing dynamic tasks, such as throwing or hitting, are referred as the kinetic chains. ${ }^{[3,4]}$ By being located in the middle of the kinetic chain system as a box, with the abdominals in the front, paraspinals and gluteals in the back, the diaphragm as the roof, and the pelvic floor and hip girdle musculature at the bottom, the core region functions as a muscular corset where forces are generated and transferred to the extremities. ${ }^{[4-6]}$ Optimal functioning of the core, leading to optimal functioning of the kinetic chain system is required for the production of strong, functional movements of the extremities. ${ }^{[7]}$

The aim of core stabilization training is to ensure appropriate muscular balance around the lumbo-pelvic-hip complex, creating a rigid cylinder against body perturbations, while allowing a stable base for accurate movement control. ${ }^{[7,8]}$ These exercises

Corresponding author: Tuğçe Özekli Mısırlıŏglu, MD. İstanbul Üniversitesi Cerrahpaşa Tıp Fakültesi Fiziksel Tıp ve Rehabilitasyon Anabilim Dalı, 34098 Cerrahpaşa, Fatih, İstanbul, Turkey. e-mail: tozeklim@gmail.com 
are known as neuromuscular retraining exercises which primarily focus on enhancing the recruitment of the local stabilizer (transversus abdominis and multifidus), global stabilizer (internal and external obliques), global mobilizer (rectus abdominus and iliocostalis), and load transfer (i.e., gluteus maximus, gluteus medius, hip adductors, rectus femoris, iliopsoas, trapezius, latissimus dorsi, deltoid, pectoralis major) muscles. ${ }^{[9]}$ A typical core stabilization program starts with abdominal drawing-in (abdominal hollowing) or abdominal bracing (co-contraction) exercises to improve neuromuscular control of the local stabilizers, moves to closed-chain segmental control exercises to further improve neuromuscular control and joint stabilization, and progresses to open kinetic chain exercises which promote distal mobility. ${ }^{[9]}$

The relationship between shoulder and core has been shown by the activation of core musculature during upper extremity movements in previous studies. ${ }^{[10-13]}$ Although the inclusion of core stabilization exercises into rehabilitation program for shoulder injury has been advocated, ${ }^{[14,15]}$ the evidence to show direct effect of core stabilization on shoulder muscle strength is still lacking. Therefore, the primary aim of the present study was to evaluate the effect of core stabilization exercise program on shoulder maximal voluntary isometric contraction (MVIC) strength. The secondary aim was to explore the immediate effect of core muscles' activation on the shoulder MVIC strength. Our primary hypothesis was that core stabilization exercises would positively affect the shoulder MVIC strength in both relaxed and core muscles activated conditions and, secondarily, the shoulder MVIC strength values would increase by voluntarily contraction of core musculature.

\section{PATIENTS AND METHODS}

This prospective, longitudinal study with a matched control group was to investigate the effect of core stabilization on shoulder muscle strength. A written informed consent was obtained from each participant. The study protocol was approved by the Koç University School of Medicine Ethics Committee. The study was conducted in accordance with the principles of the Declaration of Helsinki.

Between November 2016 and January 2015, a total of 75 consecutive healthy female volunteers (mean age 25.36 years; range, 18 to 30 years) who responded to our institutional study announcement and fulfilled the inclusion criteria were included. The inclusion criteria were as follows: female sex between the ages of
18 and 30 years, a Body Mass Index (BMI) lower than $30 \mathrm{~kg} / \mathrm{m}^{2}$, a negative upper extremity and/or spinal history of pain and/or surgery and a negative regular exercise history. The volunteers who experienced pain during the measurements or did not complete the regimen or received additional exercise interventions during the trial were excluded from the study. Of these, 42 consecutive volunteers were assigned as the study group, while the remaining 33 age- and BMI-matched volunteers were assigned as the control group. The sample sizes were determined according to the statistical calculation.

Demographic data and medical history were obtained from all participants prior to measurements. Two measurements were performed on both groups with six-week interval. Each measurement was performed by an electronic dynamometer as a set of three repetitions for each shoulder side (dominant and non-dominant) in the same position under two different conditions: core muscles relaxed and activated.

The participants were instructed how to contract their abdominals and gluteals simultaneously to achieve core muscle activation prior to measurements. The physician checked the efficacy of the contractions by palpating the abdominal and gluteal muscles. During the core muscles activated measurements, the participants were asked to start contracting their muscles two-sec before and maintain the contracted condition, until the end of each pushing.

After the first measurement, both groups were instructed to do their routine activities and not to be involved in a sports program. Those who received an exercise intervention or started to a new sports program in six-week period were not included in the second measurements.

Participants of the study group underwent a face-to-face training session for core stabilization exercises by a physiatrist after the baseline measurement. During the training session, the physiatrist checked the efficacy of each exercise by palpating the contraction of the transversus abdominis and lumbar multifidus. Exercise instruction sheets containing the illustrations with detailed descriptions of the exercises and exercise checklist sheets to be filled daily by the participants after completing their daily program was also given. Furthermore, the attendance to the exercises was checked on phone at the end of every week by the researchers. The participants were asked to attend their scheduled exercise program for at least four days in a week for at least six weeks to be considered 
compliant with the exercise schedule. Those who completed the six week core stabilization home-based exercise program successfully were preceded to the second measurement. At the second measurement, the physicians did not have access to the data from the first measurements.

\section{Testing procedures}

An electronic dynamometer (Mecmesin Myometer, West Sussex, UK) with an adjustable strap was utilized for the shoulder muscle strength measurements. Strap of the device was positioned at the wrist during the test. The MVIC applied during the test was recorded in real-time with a connected computer in Newton.

All measurements were performed by the same two physicians in a standardized protocol. The standardized test position was standing with the arm in $90^{\circ}$ of abduction in the scapular plane, the elbow extended and the forearm pronated (Figure 1). Volunteers were barefoot on a hard surface, both ankles touching each other during the test. Pushing maximally upward for five-sec, three times consecutively with one-min intervals for each shoulder side were instructed. The timings of the pushing, relaxation periods and activation of core muscles were provided by a visual timer placed in front of the participants. A loud verbal encouragement (ready 5-4-3-2-1, push, push, push) was also given throughout the measurements. Within each test series of three repetitions, the participants were instructed to rest their arm at the side between

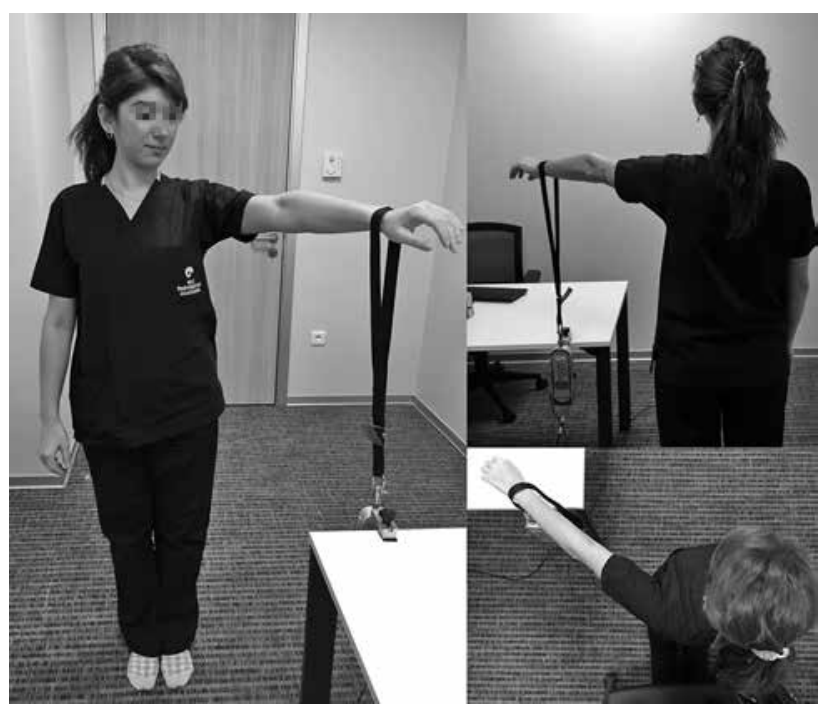

Figure 1. The participants were barefoot, both ankles touching each other. The measurements were performed in standing position with the arm in $90^{\circ}$ of abduction in the scapular plane, the elbow extended and the forearm pronated. each repetition for one-min while maintaining the feet position. Right and left shoulders were alternated between relaxed and core muscles activated conditions to prevent early fatigue.

\section{Exercise procedures}

The exercise program consisted of basic core stabilization exercises; abdominal drawing-in, abdominal drawing-in with alternating upper extremity movement, abdominal drawing-in with alternating lower extremity movement, abdominal drawing-in with alternating upper and lower extremity movement, opposite arm leg raises in prone position, pelvic tilt while standing and forward lunge (Figure 2).

\section{Exercises instructions were as follows:}

1. Abdominal drawing-in: The participants were asked to pull their navel deeply to the lumbar region and maintain the contraction for five-sec while keeping breathing lightly in supine hook-lying position. Ten repetitions with a five-sec rest between the repetitions was the target dose for this exercise.

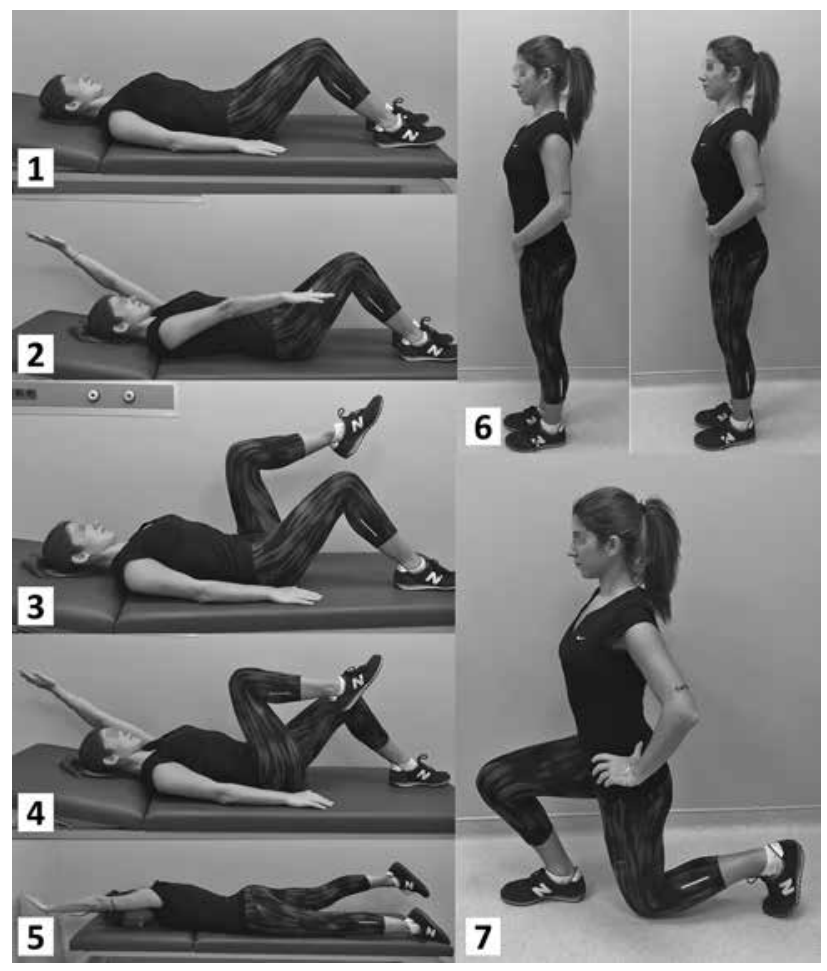

Figure 2. Basic core stabilization exercises: (1) abdominal drawing-in, (2) abdominal drawing-in with alternating upper extremity movement, (3) abdominal drawing-in with alternating lower extremity movement, (4) abdominal drawing-in with alternating upper and lower extremity movement, (5) opposite arm leg raises in prone position, (6) pelvic tilt while standing, (7) forward lunge. 
2. Abdominal drawing-in with alternating upper extremity movement: The participants were asked to flex their shoulders in forearms pronated position and return to neutral position alternatingly for five times while maintaining the abdominal contraction. The target dose for this exercise was 10 sets of five repetitions with a five-sec rest between the sets.

3. Abdominal drawing-in with alternating lower extremity movement: The participants were asked to flex the hip joint and return to neutral position alternatingly for five times while maintaining the knee joint flexion at 90 degrees and abdominal contraction. The target dose for this exercise was 10 sets of five repetitions with a five-sec rest between the sets.

4. Abdominal drawing-in with alternating upper and lower extremity movement: The participants were asked to flex their contralateral upper and lower extremities and return to neutral position alternatingly for five times while maintaining the abdominal contraction in supine position. The target dose for this exercise was 10 sets of five repetitions with a five-sec rest between the sets.

5. Opposite arm leg raises in prone position: The participants were asked to lift their contralateral upper and lower extremities to the horizontal plane alternatingly for five times while maintaining the abdominal contraction in supine position. The target dose for this exercise was 10 sets of five repetitions with a five-sec rest between the sets.

6. Pelvic tilt while standing: The participants were asked to contract their gluteal and abdominal muscles to rotate their hips in a posterior direction and maintain the contraction for five-sec while keeping breathing lightly in standing position. Ten repetitions with a five$\mathrm{sec}$ rest between the repetitions was the target dose for this exercise.
7. Forward lunge: The participants were asked to step forward, flex the hip as far as the contralateral knee touches slightly to the floor and, then, push off the front leg and return to the starting position. Emphasis was placed on maintaining a neutral spinal posture, contracting the abdominal muscles, and breathing normally throughout the exercise. Tens sets of five repetitions for each side with a five-sec rest between the sets was the target dose for this exercise.

\section{Statistical analysis}

Statistical analysis was performed with the Number Cruncher Statistical System (NCSS) 2007 statistical software (NCSS LLC, Kaysville, Utah, USA) and MedCalc Statistical Software Version 12.5 (MedCalc Software, Ostend, Belgium). In addition to descriptive statistical methods (mean, standard deviation [SD]), unpaired and paired Student t-tests were used. The Shapiro-Wilk test was used to assess normal distribution. Intraclass correlation was used to assess test reliability. Intraclass correlation coefficient (ICC) level of greater than 0.700 was accepted as reliable. A $p$ value of $<0.05$ was considered statistically significant with $95 \%$ confidence interval (CI).

Due to missing data in the literature regarding the effect of core stabilization exercises on the shoulder MVIC strength, we were unable to perform a power analysis prior to the study. Therefore, a post-hoc analysis was performed. ${ }^{[16]}$

\section{RESULTS}

The number of eligible volunteers included in the baseline measurements was 42 in the study group and 33 in the control group. As the adherence of nine participants to the exercise program was considered low and three participants from the control group refused to take place in the second measurements, statistical analysis was performed on the remaining 33 participants from the study and 30 participants from the control group.

Table 1. Demographic characteristics of the participants

\begin{tabular}{lcccc}
\hline & Study group $(\mathrm{n}=33)$ & & Control group $(\mathrm{n}=30)$ & \\
\cline { 2 - 2 } Characteristics & Mean $\pm \mathrm{SD}$ & & Mean $\pm \mathrm{SD}$ & \\
\hline Age (year) & $25.97 \pm 2.65$ & & $24.87 \pm 3.87$ & 0.19 \\
Height (meters) & $1.62 \pm 0.06$ & & $1.64 \pm 0.06$ & 0.43 \\
Weight (kilograms) & $58.45 \pm 10.44$ & & $56.92 \pm 8.39$ & 0.52 \\
Body Mass Index $\left(\mathrm{kg} / \mathrm{m}^{2}\right)$ & $22.1 \pm 3.39$ & & $21.22 \pm 2.77$ & 0.26 \\
\hline SD: Standard deviation; Student's t-test was used to compare age, height, weight and Body Mass Index; $\mathrm{p}<0.05$.
\end{tabular}


Table 2. Intraclass Correlation Coefficients of all the measurements

\begin{tabular}{llccc}
\hline & \multicolumn{1}{c}{ Position } & ICC & \multicolumn{2}{c}{$95 \%$ Confidence interval } \\
\cline { 4 - 5 } Dominancy & Relaxed & 0.954 & 0.938 & 0.966 \\
Dominant side & Core muscles activated & 0.976 & 0.968 & 0.983 \\
\multirow{2}{*}{ Non-dominant side } & Relaxed & 0.973 & 0.964 & 0.981 \\
& Core muscles activated & 0.978 & 0.971 & 0.984 \\
\hline ICC: Intraclass Correlation Coefficient; Intraclass correlation was used to assess test reliability; ${ }^{*}$ Accepted level of ICC $>0.700$.
\end{tabular}

Demographic data of both groups including age, height, weight, and BMI are given in Table 1 . There was no significant difference between the groups in terms of these parameters. Intraclass correlation coefficients of all the measurements were above the accepted level of 0.700 (Table 2). All data sets were distributed normally.

The shoulder MVIC strength of both groups at baseline and six weeks in relaxed and core muscles contracted conditions for the dominant and nondominant sides are given in Table 3 . There was no statistically significant difference in the MVIC strength values between two groups at baseline for both sides and conditions. However, a statistically significant difference existed between the groups at six week measurements. The MVIC strength values at six weeks were significantly higher in the study group.

To investigate the effect of core muscles' activation on shoulder muscle strength, we compared the MVIC strength of dominant and non-dominant sides in relaxed conditions with the strengths in core muscles activated conditions in both groups. The MVIC strengths in core muscles activated condition were significantly lower than strengths in relaxed conditions for both groups at baseline and six-week measurements (Table 3).

The effect of core muscle stabilization exercise program on shoulder muscle strength was investigated by comparing the measurement values of the study group at baseline and six weeks. These comparisons were carried out for dominant and non-dominant sides, relaxed and core muscles activated conditions. The MVIC strength values after a six-week core stabilization program were significantly higher in all aforementioned conditions. On the other hand, there was a significant decrease in all MVIC strength values of the control group at six-week measurements (Figure 3).

The post-hoc power values of the tests at six weeks were above $80 \%$, which was the adequate limit for statistical significance. These values were $96.5 \%$ for the dominant side in relaxed position; $85.2 \%$ for the dominant side in core muscles activated position; 93.2\% for the non-dominant side in relaxed position, and $92.5 \%$ for the non-dominant side in core muscles activated position.

Table 3. Mean shoulder muscle strength values between the groups according to dominancy and position at baseline and six-week measurements

\begin{tabular}{|c|c|c|c|c|c|}
\hline \multirow[b]{2}{*}{ Measurement } & \multirow[b]{2}{*}{ Dominancy } & \multirow[b]{2}{*}{ Position } & \multirow{2}{*}{$\begin{array}{c}\text { Study group } \\
\text { Mean } \pm \text { SD }\end{array}$} & \multirow{2}{*}{$\begin{array}{c}\text { Control group } \\
\text { Mean } \pm \text { SD }\end{array}$} & \multirow[b]{2}{*}{$p$} \\
\hline & & & & & \\
\hline \multirow[t]{6}{*}{ Baseline } & Dominant side & Relaxed & $60.75 \pm 13.07$ & $62.95 \pm 11.28$ & $0.47 \varepsilon$ \\
\hline & & Core muscles activated & $56.98 \pm 11.51$ & $59.84 \pm 10.67$ & $0.31 \varepsilon$ \\
\hline & & $p$ & $0.0013^{\star} \dagger$ & $<0.001^{\star} \dagger$ & \\
\hline & Non-dominant side & Relaxed & $57.69 \pm 11.41$ & $59.47 \pm 11.44$ & $0.53 \varepsilon$ \\
\hline & & Core muscles activated & $55.47 \pm 11.45$ & $56.20 \pm 9.51$ & $0.78 \varepsilon$ \\
\hline & & $p$ & $0.01^{\star} \dagger$ & $<0.001^{\star} \dagger$ & \\
\hline \multirow[t]{6}{*}{$6^{\text {th }}$ week } & Dominant side & Relaxed & $68.67 \pm 11.06$ & $56.96 \pm 13.36$ & $<0.001^{\star} \varepsilon$ \\
\hline & & Core muscles activated & $64.10 \pm 10.54$ & $54.90 \pm 13.43$ & $0.003^{\star} \varepsilon$ \\
\hline & & $p$ & $<0.001^{\star} \dagger$ & $0.02^{\star} \dagger$ & \\
\hline & Non-dominant side & Relaxed & $65.38 \pm 9.84$ & $55.55 \pm 12.45$ & $<0.001^{\star} \varepsilon$ \\
\hline & & Core muscles activated & $61.65 \pm 9.32$ & $52.45 \pm 11.85$ & $0.0011^{\star} \varepsilon$ \\
\hline & & $p$ & $<0.001^{\star} \dagger$ & $<0.001^{\star} \dagger$ & \\
\hline
\end{tabular}

SD: Standard deviation; Student's t-test was used for comparisons; $\dagger$ Indicates paired; $\varepsilon$ Indicates unpaired t-test; ${ }^{*} \mathrm{P}<0.05$ indicates statistically significant difference. 


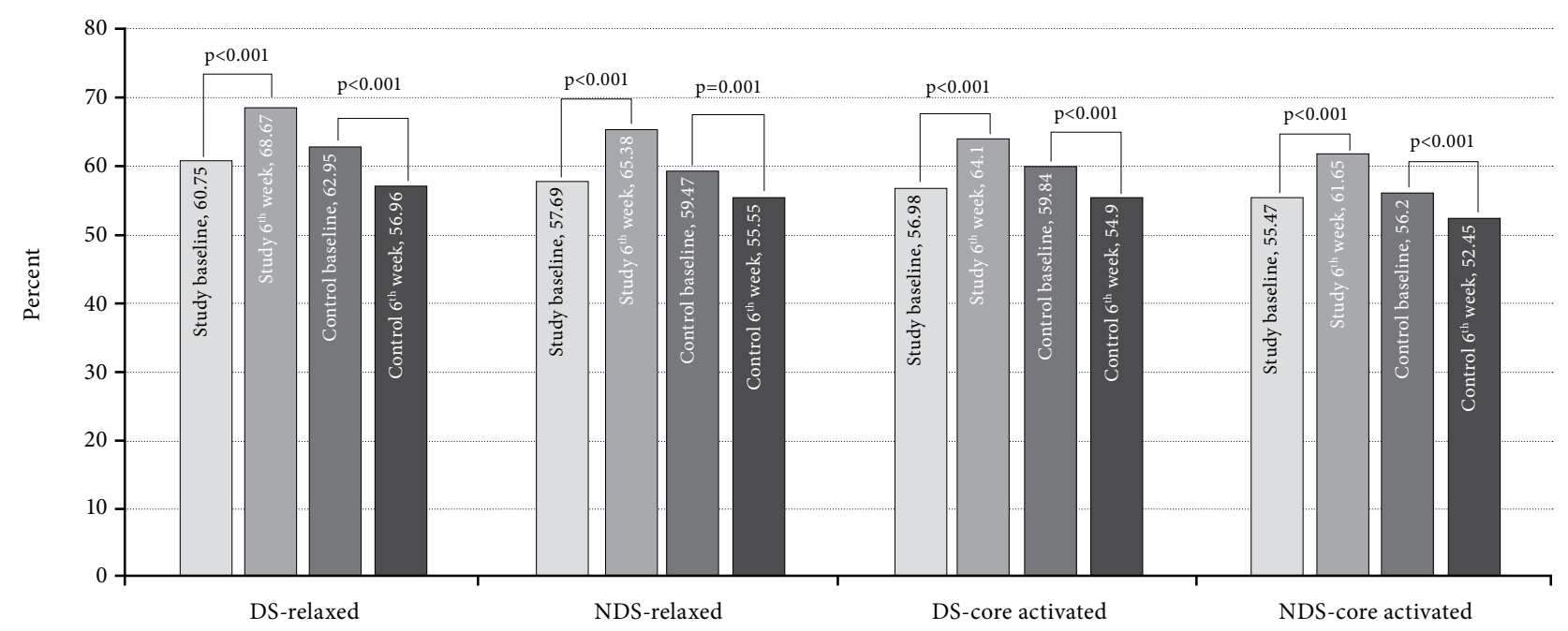

Figure 3. Bar graph showing mean shoulder strength values according to dominancy and position at baseline and six-week measurements.

\section{DISCUSSION}

A proximal stability approach, also named as kinetic chain approach to shoulder rehabilitation, was commonly mentioned in the literature by Kibler and McMullen. ${ }^{[2,17,18]}$ This protocol mainly focuses on using distal segments, such as trunk and legs to drive the scapula and shoulder during the rehabilitation process. The scapula stabilization via kinetic chain exercises has been proposed to achieve an effective and efficient rotator cuff strengthening. ${ }^{[2]}$ To promote a properly functioning kinetic chain system, a properly functioning core musculature has been advocated. ${ }^{[7,14,19]}$ Based on these hypotheses, we evaluated the effect of core stabilization on the shoulder muscle strength.

Shoulder strength testing method used in this study was performed according to the test protocol described by Constant et al. ${ }^{[20]}$ The maximum strength value of three repetitions, each separated by at least one-min and performed at $90^{\circ}$ of shoulder abduction in the scapular plane with the elbow in pronation were found to reflect best function in real terms, previously. Therefore, we preferred using a maximum value rather than a mean value as advocated by Constant et al. ${ }^{[20]}$ In this study, all MVIC measurements performed were considered as reliable (ICC $>0.90)$.

The results of the current study suggested that a six-week core stabilization home-based exercise program might cause a significant increase in the shoulder MVIC strength in healthy individuals. This finding is also supported by previous studies reporting a significant relationship between core stability and shoulder. The influence of shoulder exercises on core stability was well-documented in literature. ${ }^{[21-23]}$ It was shown that upper limb movements in different directions performed in the standing or sitting position produced varying degrees of activities in abdominal and back muscles. ${ }^{[21,22]}$ In a study by Tarnanen, ${ }^{[10]}$ of all the isometric shoulder exercises, unilateral horizontal shoulder abduction and bilateral shoulder extension while standing were found to be associated with the highest activation of core musculature, namely multifidus-longissimus, and external oblique-rectus abdominis muscles, respectively. In addition, it was shown that shoulder resistance exercises could increase the endurance and strength of core stability muscles, when the pelvis was supported. ${ }^{[10,24]}$ On the other hand, the number of studies investigating the effects of core stability on the upper extremity is limited. Most of these studies examined the effects of core strength/stability on sport-specific performance such as swimming, rowing, running, throwing and were unable to establish a consistent relationship. ${ }^{[25-28]}$

Several core stability training programs aimed at reducing back pain and preventing injury have been proposed in the literature. The exercises which we selected in this study constituted simple, beginning core exercises with low load and high repetitions, or holding time. The main goal of our exercise selection was based on the concept of neuromuscular retraining of the core rather than its pure strengthening. ${ }^{[5,29,30]}$ Our aim was to improve core stability by improving the ability of the participant's control over the core region. ${ }^{[19]}$ As in all other core stability training 
programs, the first exercise was abdominal drawingin, which was known to recruit both the oblique and transversus abdominis muscles. ${ }^{[31]}$ The other exercises aimed to provide proper lumbopelvic control, while adding dynamic upper and lower extremity movements to improve muscle recruitment and distal stability.

Since core stability is a complex interaction among local, global, and load transfer muscles, accurate assessment of core stability is also complex and challenging. Although many tests to measure core stability have been described, these tests often measure one aspect of core stability, such as muscle recruitment, muscle strength and endurance, postural control, balance, and movement patterns. ${ }^{[7,32-36]}$ Due to the lack of consensus on how to measure core stability, the effectiveness of our exercise program in the study group was unable to be assessed. However, the ability of the participant's control over the core region was checked at the time of exercise training by the palpation method. Previous studies also showed that only low-level activation, $<10 \%$ of the MVIC, of transversus abdominis was required for the drawing-in maneuver. ${ }^{[30,37,38]}$

A secondary finding of interest in this study was the significant decrease in shoulder MVIC strength values by activation of core muscles by self-contracting abdominals and gluteals actively. This result was inconsistent with our hypothesis and existing literature, demonstrating the positive relation between shoulder and core muscles. ${ }^{[39,40]}$ In a study by Cacolice et al., ${ }^{[39]}$ the effect of volitional isometric contraction of the abdominals on isometric shoulder flexion torque on healthy individuals was studied. It was found that isometric shoulder flexion torque values at 90 degrees of flexion were greater, when an individual volitionally superimposed a contraction of the abdominals compared to torque values, when the abdominals were solely recruited in a subconscious fashion. The difference in results between this study and ours may be attributed to the difference in the strength measurement techniques (isokinetic versus isometric), the planes of the shoulder strengths measured (flexion versus scapular abduction), and the extent of core muscle activations (abdominals versus abdominals and gluteals). In another study, Rosemeyer et al. ${ }^{[40]}$ investigated the effect of core musculature fatigue on maximum shoulder strength. They conducted a corefatiguing protocol which included endurance-hold core-fatigue tests performed in flexion and extension postures. The MVIC strength measurements were performed by a handheld dynamometer immediately before and after the protocol. The results indicated a significant decrease in shoulder MVIC strength values in the frontal and transvers planes, and a significant decrease in the first and second strength test measurements regardless of the strength test measured. Although there seems to be an inverse relationship between the results of this study and ours at first glance, two studies are totally different. In the aforementioned study, it is unclear if the fatiguing protocol makes the core region weaker, instable or just dysfunctional. Besides, by contracting the core musculature volitionally, it is unclear whether we are creating a more stable core. Therefore, the results of these studies should be discussed separately.

On the other hand, our result may resemble in some way the study done by Sato et al. ${ }^{[4]}$ They investigated the effects of teeth clenching on shoulder abduction. It was observed that voluntary teeth clenching suppressed motor unit recruitment during isometric muscle contractions of arm abduction. Presumably, via not the same mechanism with the mentioned study, our results suggest that self-activation of core muscles during the shoulder strength measurement have an inhibitory effect on the MVIC strength. However, further studies are needed to elucidate the physiological mechanism underlying this possible inhibitory effect.

At the six-week measurements, while there was a significant improvement in all MVIC strength values in the study group, a significant decrease was conversely observed in the measurements of the control group. This result seems a bit conflicting, as the control group was instructed to continue their daily routine activities during the six-week study interval and change in shoulder muscle strength was not an expected finding. However, it is often accepted that a six-week period is long enough for a muscle to gain or lose strength. ${ }^{[42]}$ Therefore, studies about test-retest reliability of muscle strength tests commonly prefer using shorter time gaps between the measurements. There is no information in the literature regarding change in muscle strength of healthy individuals with this long interval. In the present study, we investigated whether there was a change in the shoulder MVIC strength after receiving a core stabilization exercise program. Our results supported the positive effect of core stabilization exercise program on the shoulder MVIC strength. However, the significant change in the shoulder strength of the control group highlights the need for further studies on change in the muscle strength of healthy individuals with long intervals. 
Nonetheless, there are some limitations to this study. First, this study was carried out on a limited population of young healthy female individuals. Therefore, further studies are needed to analyze whether core stabilization exercises are effective on shoulder muscle strength in the elderly and/or male sex and in patients with shoulder pathologies. Second, our study and control groups were matched, but non-randomized. Finally, our hypothesis that our exercise program was effective in increasing core stability was based on previous studies. ${ }^{[3,21,22,32,43]}$ Since the main target of core stabilization exercises are neuromuscular retraining of the core rather than its pure strengthening ${ }^{[5,19]}$ and there is no direct method to evaluate core stability, we were unable to determine the effectiveness of our exercises on core stability in the study group.

The main findings of our study were as follows:

1. The activation of core muscles by selfcontracting abdominals and gluteals actively during the shoulder strength measurement significantly decreased the MVIC strength values, regardless of hand dominancy and time of the measurement (before or after six-week core stabilization exercise program)

2. The MVIC strength values significantly increased after six-week core stabilization home-based exercise program on both shoulder sides and in both conditions (i.e., relaxed or core muscle activated).

In conclusion, our results support the use of core stabilization exercises in the early periods of shoulder rehabilitation, when the shoulder muscle strengthening exercises are painful and/or shoulder immobilization is necessary. In addition, to the best of our knowledge, this is the first study to investigate the effect of core muscles activated posture on shoulder abduction strength measurement. Inconsistent with our hypothesis, the activation of core muscles by selfcontracting abdominals and gluteals actively during the shoulder abduction strength measurement significantly decreased the maximum shoulder abduction strength values. This finding suggests us the importance of standardization of core muscles activated or relaxed posture during the shoulder strength measurements. Additional studies should investigate the effect of core stabilization on shoulder muscle strength in patients with different shoulder pathologies.

\section{Declaration of conflicting interests}

The authors declared no conflicts of interest with respect to the authorship and/or publication of this article.

\section{Funding}

The authors received no financial support for the research and/or authorship of this article.

\section{REFERENCES}

1. Moghadam AN, Mohammadi R, Arab AM, Kazamnajad A. The effect of shoulder core exercises on isometric torque of glenohumeral joint movements in healthy young females. J Res Med Sci 2011;16:1555-63.

2. McMullen J, Uhl TL. A kinetic chain approach for shoulder rehabilitation. J Athl Train. 2000;35:329-37.

3. Akuthota V, Ferreiro A, Moore T, Fredericson M. Core stability exercise principles. Curr Sports Med Rep 2008;7:39-44.

4. Vleeming A, Pool-Goudzwaard AL, Stoeckart R, van Wingerden JP, Snijders CJ. The posterior layer of the thoracolumbar fascia. Its function in load transfer from spine to legs. Spine (Phila Pa 1976) 1995;20:753-8.

5. Akuthota V, Nadler SF. Core strengthening. Arch Phys Med Rehabil 2004;85:86-92.

6. Sciascia A, Cromwell R. Kinetic chain rehabilitation: a theoretical framework. Rehabil Res Pract 2012;2012:853037.

7. Kibler WB, Press J, Sciascia A. The role of core stability in athletic function. Sports Med 2006;36:189-98.

8. Panjabi MM. The stabilizing system of the spine. Part II. Neutral zone and instability hypothesis. J Spinal Disord 1992;5:390-6.

9. Brumitt J, Matheson JW, Meira EP. Core stabilization exercise prescription, part I: current concepts in assessment and intervention. Sports Health 2013;5:504-9.

10. Tarnanen SP, Ylinen JJ, Siekkinen KM, Mälkiä EA, Kautiainen HJ, Häkkinen AH. Effect of isometric upperextremity exercises on the activation of core stabilizing muscles. Arch Phys Med Rehabil 2008;89:513-21.

11. Moreside JM, Vera-Garcia FJ, McGill SM. Trunk muscle activation patterns, lumbar compressive forces, and spine stability when using the bodyblade. Phys Ther 2007;87:153-63.

12. Hodges PW, Richardson CA. Relationship between limb movement speed and associated contraction of the trunk muscles. Ergonomics 1997;40:1220-30.

13. Hodges PW, Richardson CA. Feedforward contraction of transversus abdominis is not influenced by the direction of arm movement. Exp Brain Res 1997;114:362-70.

14. Brumitt J, Dale RB. Integrating shoulder and core exercises when rehabilitating athletes performing overhead activities. N Am J Sports Phys Ther 2009;4:132-8.

15. Radwan A, Francis J, Green A, Kahl E, Maciurzynski D, Quartulli A, et al. Is there a relation between shoulder dysfunction and core instability: Int J Sports Phys Ther 2014;9:8-13.

16. Rosner B. Fundamentals of Biostatistics. 7th ed. Boston: Cengage Learning; 2011.

17. Kibler WB, McMullen J, Uhl T. Shoulder rehabilitation strategies, guidelines, and practice. Orthop Clin North Am 2001;32:527-38.

18. Kibler WB. The role of the scapula in athletic shoulder function. Am J Sports Med 1998;26:325-37. 
19. Miyake Y, Kobayashi R, Kelepecz D, Nakajima M. Core exercises elevate trunk stability to facilitate skilled motor behavior of the upper extremities. J Bodyw Mov Ther 2013;17:259-65.

20. Constant CR, Gerber C, Emery RJ, Søjbjerg JO, Gohlke F, Boileau P. A review of the Constant score: modifications and guidelines for its use. J Shoulder Elbow Surg 2008;17:355-61.

21. Arokoski JP, Valta T, Airaksinen O, Kankaanpää M. Back and abdominal muscle function during stabilization exercises. Arch Phys Med Rehabil 2001;82:1089-98.

22. Arokoski JP, Kankaanpää M, Valta T, Juvonen I, Partanen J, Taimela $S$, et al. Back and hip extensor muscle function during therapeutic exercises. Arch Phys Med Rehabil 1999;80:842-50.

23. Behm DG, Leonard AM, Young WB, Bonsey WA, MacKinnon SN. Trunk muscle electromyographic activity with unstable and unilateral exercises. J Strength Cond Res 2005;19:193-201.

24. Tarnanen SP, Siekkinen KM, Häkkinen AH, Mälkiä EA, Kautiainen HJ, Ylinen JJ. Core muscle activation during dynamic upper limb exercises in women. J Strength Cond Res 2012;26:3217-24.

25. Scibek JS, Guskiewicz KM, Prentice WE, Mays S, Davis JM. The Effect of Core Stabilization Training on Functional Performance in Swimming. Chapel Hill: Master's Thesis, University of North Carolina; 2001.

26. Stanton R, Reaburn PR, Humphries B. The effect of shortterm Swiss ball training on core stability and running economy. J Strength Cond Res 2004;18:522-8.

27. Tse MA, McManus AM, Masters RS. Development and validation of a core endurance intervention program: implications for performance in college-age rowers. J Strength Cond Res 2005;19:547-52.

28. Saeterbakken AH, van den Tillaar R, Seiler S. Effect of core stability training on throwing velocity in female handball players. J Strength Cond Res 2011;25:712-8.

29. Ebenbichler GR, Oddsson LI, Kollmitzer J, Erim Z. Sensory-motor control of the lower back: implications for rehabilitation. Med Sci Sports Exerc 2001;33:1889-98.

30. Richardson C, Jull G, Hodges PW, Hides J. Therapeutic
Exercise for Spinal Segmental Stabilization in Low Back Pain: Scientific Basis and Clinical Approach. 1st ed. Edinburgh: Churchill Livingstone; 1999.

31. Sahrmann SA. Diagnosis and Treatment of Movement System Impairments, St. Loius: Mosby Inc.; 2002.

32. Huxel Bliven KC, Anderson BE. Core stability training for injury prevention. Sports Health 2013;5:514-22.

33. Escamilla RF, Lewis C, Bell D, Bramblet G, Daffron J, Lambert $S$, et al. Core muscle activation during Swiss ball and traditional abdominal exercises. J Orthop Sports Phys Ther 2010;40:265-76.

34. Liemohn WP, Baumgartner TA, Gagnon LH. Measuring core stability. J Strength Cond Res 2005;19:583-6.

35. McGill SM, Childs A, Liebenson C. Endurance times for low back stabilization exercises: clinical targets for testing and training from a normal database. Arch Phys Med Rehabil 1999;80:941-4.

36. Minick KI, Kiesel KB, Burton L, Taylor A, Plisky P, Butler RJ. Interrater reliability of the functional movement screen. J Strength Cond Res 2010;24:479-86.

37. McGill S. Low Back Disorders: Evidence-based Prevention and Rehabilitation. Champaign IL: Human Kinetics; 2002.

38. Richardson C, Hodges P, Hides J. Therapeutic Exercise for Lumbopelvic Stabilization. A Motor Control Approach for the Treatment and Prevention of Low Back Pain. 2nd ed. London: Churchill Livingstone; 2004.

39. Cacolice PA, Carcia CR, Scibek JS. Shoulder Flexion Torque Is Augmented By A Volitional Abdominal Contraction. J Athl Training 2013;48:S-155.

40. Rosemeyer JR, Hayes BT, Switzler CL, Hicks-Little CA. Effects of Core-Musculature Fatigue on Maximal Shoulder Strength. J Sport Rehabil 2015;24:384-90.

41. Sato H, Kawano T, Saito M, Toyoda H, Maeda Y, Türker $\mathrm{KS}$, et al. Teeth clenching reduces arm abduction force. Exp Brain Res 2014;232:2281-91.

42. Hall JE. Sports Physiology. In: Hall JE, editor. Guyton and Hall Textbook of Medical Physiology. Philadelphia: Elsevier Inc.; 2016.

43. Brumitt J, Matheson JW, Meira EP. Core stabilization exercise prescription, part I: current concepts in assessment and intervention. Sports Health 2013;5:504-9. 